SISSA 18/2003/EP

$\mathrm{TIFR} / \mathrm{TH} / 03-05$

hep-ph/0303078

\title{
CP violation and matter effect for a variable earth density in very long baseline experiments
}

\author{
Biswajoy Brahmachari ${ }^{a, d}$, Sandhya Choubey ${ }^{b}$ and Probir Roy ${ }^{c}$ \\ a Theory Group, Saha Institute of Nuclear Physics, \\ AF/1 Bidhannagar, Kolkata 700 064, India \\ ${ }^{b}$ INFN, Sezione di Trieste and Scuola Internazionale Superiore di Studi Avanzati, \\ I-34014, Trieste, Italy \\ $c$ Tata Institute of Fundamental Research, \\ Homi Bhaba Road, Mumbai 400 005, India \\ ${ }^{d}$ Department of Physics, Vidyasagar Evening College, \\ 39, Sankar Ghosh Lane, Kolkata 700 006, India
}

\begin{abstract}
$\underline{\text { Abstract }}$
The perturbative treatment of subdominant oscillation and matter effect in neutrino beams/superbeams, propagating over long baselines and being used to look for CP violation, is studied here for a general matter density function varying with distance. New lowest order analytic expressions are given for different flavour transition and survival probabilities in a general neutrino mixing basis and a variable earth matter density profile. It is demonstrated that the matter effect in the muon neutrino (antineutrino) flavour survival probability vanishes to this order, provided the depletion, observed for atmospheric muon neutrinos and antineutrinos at super-Kamiokande, is strictly maximal. This result is independent of the earth density profile and the distance L between the source and the detector. In the general variable density case we show that one cannot separate the matter induced asymmetry from a genuine CP effect by keeping two detectors at distances $L_{1}$ and $L_{2}$ from the source while maintaining a fixed ratio $L_{1} / E_{1}=L_{2} / E_{2}$. This needs to be done numerically and we estimate the asymmetry generated by the earth matter effect with particular density profiles and some chosen parameters for very long baseline neutrino oscillation experiments.
\end{abstract}




\section{Introduction}

Finding $\mathrm{CP}$ violation in the neutrino sector [1] is a tantalizing goal waiting to be attained [2] in forthcoming long baseline experiments with neutrino beams/superbeams and future ones at neutrino factories. The discovery and quantitative measurement of such an effect will not only open a new window for physics beyond the standard model, but may provide an insight into leptogenesis [3]. From solar neutrino studies and the reactor experiment by KAMLAND, we already know [4] the squared mass difference and the angle of mixing between concerned mass eigenstate pair $\nu_{1,2}$ to be $\Delta m_{21}^{2} \equiv m_{2}^{2}-m_{1}^{2} \sim 7 \times 10^{-5} \mathrm{eV}^{2}$ and $\sin ^{2} \theta_{12} \sim 0.3$ respectively (see [5] and references therein). Similarly, atmospheric neutrino studies [6] have revealed the corresponding parameters for the other mass eigenstate pair $\nu_{2,3}$ to be $\left|\Delta m_{32}^{2}\right| \equiv\left|m_{3}^{2}-m_{2}^{2}\right| \sim 3 \times 10^{-3} \mathrm{eV}^{2}$ and $\sin ^{2} \theta_{23} \sim 0.5$. Other reactor experiments [7] have, however, shown that the third possible mixing angle $\theta_{13}$ is very small, consistent with zero. These mysterious numbers have increased the importance of the task of getting a quantitative handle on $\mathrm{CP}$ violation likely to be associated with the MNS neutrino mixing matrix. This is sought $[1,2]$ to be achieved by measuring the difference between neutrino and antineutrino beams in the muonic to electronic flavour transition probability which can be obtained from the general expression

$$
\Delta P_{\beta \alpha}(L, E)=P\left[\nu_{\alpha}(0) \rightarrow \nu_{\beta}(L)\right]-P\left[\bar{\nu}_{\alpha}(0) \rightarrow \bar{\nu}_{\beta}(L)\right]
$$

over a large distance $L, E$ being the beam energy and $\alpha, \beta$ being the flavour indices. If $\left|\Delta m_{32}^{2}\right| L / E$ is chosen to be $\mathcal{O}(1)$, the effect will be driven dominantly by $\nu_{\mu}-\nu_{\tau}$ oscillation.

A major practical problem, associated with the above task, is the occurrence of the matter effect in neutrino flavour transitions studied in long baseline experiments. These contribute to $\Delta P_{\beta \alpha}(L)$ and induce a "fake CP violation". The latter needs to be filtered out, leaving only the genuine $\mathrm{CP}$ violation part. However, the matter effect is not merely a background but deserves to be studied in its own right. In particular, it can yield valuable information on the sign of $\Delta m_{32}^{2}$, on the MSW resonant enhancement of neutrino oscillations and on the mixing between neutrinos of the first and the third generations. It is important therefore to be able to compute the matter effect relevant to any given experiment looking for CP violation. Several studies [2, 9, 10, 11] have been conducted to this end. Specifically, we shall use the formalism of Arafune et al. [11] developed to treat the matter effect as well as the subdominant oscillation driven by $\Delta m_{21}^{2}$ and $\theta_{12}$ perturbatively to the lowest order relative to the dominant oscillation driven by $\left|\Delta m_{32}^{2}\right|$ and $\theta_{23}$. Thus the evolution matrix element $S_{\beta \alpha}(x) \equiv<\nu_{\beta}(x) \mid \nu_{\alpha}(0)>, \alpha, \beta$ being flavour indices, could be calculated to first order in $\Delta m_{21}^{2}$ and $a$, where

$$
a(x, E)=2 \sqrt{2} G_{F} N_{e} E=7.56 \times 10^{-5} \mathrm{eV}^{2} \frac{\mathrm{E}}{\mathrm{GeV}} \frac{\rho(\mathrm{x})}{\mathrm{gms} / \mathrm{cc}},
$$

with $N_{e}$ being the electron density and $\rho(x)$ the mass density of the earth, expressed as a function of the path length $x$ of the beam. The parameter $\rho$ was assumed in Ref. [11] to be spatially uniform $^{1}$. This is not always a realistic assumption [9], especially for future very long baseline experiments where nonuniformity in earth's density profile cannot be neglected. One possible

\footnotetext{
${ }^{1}$ For instance, one could assume [12] a constant density $\rho$ equal to the average density of the PREM profile [13].
} 
approach [14] is to write the density function $\rho(x)$, and correspondingly $a(x, E)$, as an average constant plus a spatially fluctuating part and expand the latter into Fourier modes, arguing that only the first few modes is important for foreseable experiments. There is, however, a large uncertainly $[15,16,17]$ in the seismological knowledge of the latter; indeed, this feeds back into the average density parameter. Under the circumstances, it is worthwhile making — within the lowest order perturbative framework - general statements for an arbitrarily varying $a(x, E)$ that can be checked by experimental measurements. This is our aim here. Our approach is basically analytic and the price to pay is to use lowest order perturbation theory. As will be pointed out in our section on numerical studies, a lowest order perturbative result is often invalid in very long baseline neutrino experiments. There are, however, sizable domains in the $(L, E)$ plane where it should be reliable. At least, the importance of the effects considered is clearly brought out by our analytic considerations.

In this paper we reexamine the treatment of Ref. [11], assuming an arbitrary spatial dependence in $\rho(x)$ and hence $a(x, E)$, consistent with the approximation $|a|<<\left|\Delta m_{32}^{2}\right|$. We give extended versions of the formulae of Arafune et al, accommodating such a nonuniform $\rho(x)$ and in a general neutrino mixing basis. Even with such an arbitrary earth density profile, we find that the matter effect in the muon neutrino (antineutrino) flavour survival probability $P\left[\stackrel{(-)}{\nu_{\mu}}(0) \rightarrow \stackrel{(-)}{\nu_{\mu}}(L)\right]$ vanishes if the flavour conversion of atmospheric $\nu_{\mu}$ and $\bar{\nu}_{\mu}$, as observed in super-K, is truly maximal. However, the simple methods, proposed in Ref. [11] to filter out the matter effect terms, are specific to the uniform earth density assumption and do not extend to the variable density case in the lowest order of perturbation theory. We show that, in general, it is not possible to separate the 'fake' matter induced asymmetry from the genuine CP effect by keeping two detectors at distances $L_{1}$ and $L_{2}$ from the source while keeping the ratio $L_{1} / E_{1}=L_{2} / E_{2}=L / E$ fixed and taking a linear combination of $P\left[\nu_{\mu}(0) \rightarrow \nu_{\beta}(L)\right]$ and $P\left[\bar{\nu}_{\mu}(0) \rightarrow \bar{\nu}_{\beta}(L)\right]$, as proposed for a constant matter density profile to the lowest perturbative order in subdominant oscillation and matter effects by Arafune et al. This separation has to be done numerically and in the last part we compute the asymmetries generated by the earth's matter effect for sample earth matter density profiles keeping specific experimental possibilities in mind.

This paper is organized as follows. Section 2 contains an extension of the lowest order perturbative calculation of the evolution operator to the variable density case. In Section 3 we calculate the muon (anti)-neutrino flavour survival probability $P[\stackrel{(-)}{\nu},(0) \rightarrow \stackrel{(-)}{\nu}(L)]$. This calculation is extended to the general flavour transition probability $P\left[\stackrel{(-)}{\nu_{\alpha}}(0) \rightarrow \stackrel{(-)}{\nu_{\beta}}(L)\right]$ in Section 4 . Our numerical studies are presented in Section 5. Section 6 contains a summary of our results. 


\section{Evolution operator formalism}

The formulae for the amplitude and probability of the transition of $\nu_{\alpha}(0)$ to $\nu_{\beta}(L)$ in vacuum are ${ }^{2}$

$$
\begin{aligned}
A\left(\nu_{\alpha}(0) \rightarrow \nu_{\beta}(L)\right)= & <\nu_{\beta}(L) \mid \nu_{\alpha}(0)>=\sum_{i} U_{\alpha i}^{*} e^{\frac{-m_{i}^{2} L}{2 E}} U_{\beta i}, \\
P\left(\nu_{\alpha}(0) \rightarrow \nu_{\beta}(L)\right)= & \delta_{\alpha \beta}-4 \sum_{j>i} R e\left(U_{\alpha i} U_{\beta i}^{*} U_{\alpha j}^{*} U_{\beta j}\right) \sin ^{2} \frac{\Delta m_{i j}^{2} L}{4 E} \\
& +2 \sum_{j>i} \operatorname{Im}\left(U_{\alpha i} U_{\beta i}^{*} U_{\alpha j}^{*} U_{\beta j}\right) \sin \frac{\Delta m_{i j}^{2} L}{2 E},
\end{aligned}
$$

where $\Delta m_{i j}^{2}=m_{i}^{2}-m_{j}^{2}$ and the neutrino mixing matrix $U$ is defined in vacuum by $\left|\nu_{\alpha}>=U_{\alpha i}\right| \nu_{i}>$ , $\alpha$ and $i$ being flavour and mass eigenstate indices respectively. More generally, when matter is present, one can decompose the Hamiltonian as

$$
H=H_{0}+H^{\prime}
$$

where $H_{0}$ is the unperturbed part containing $\Delta m_{32}^{2}$ while $H^{\prime}$ is the perturbation involving $\Delta m_{21}^{2}$ and $a(x, E)$.

In the case of an $x$-dependent $H^{\prime}$, we cannot use the replacement procedure of Ref. [18], but need to use the evolution operator formalism. Thus we write

$$
\left|\nu_{\beta}(x)>=S_{\beta \alpha}(x)\right| \nu_{\beta}(0)>,
$$

where the operator $S$ obeys the evolution equation

$$
i \frac{d S}{d x}=H_{0} S(x)+H^{\prime}(x) S(x)
$$

with

$$
\begin{aligned}
H_{0} & =\frac{1}{2 E} U\left(\begin{array}{ccc}
0 & 0 & 0 \\
0 & 0 & 0 \\
0 & 0 & \Delta m_{32}^{2}
\end{array}\right) U^{\dagger}, \\
H^{\prime}(x) & =\frac{1}{2 E}\left[U\left(\begin{array}{ccc}
0 & 0 & 0 \\
0 & \Delta m_{21}^{2} & 0 \\
0 & 0 & 0
\end{array}\right) U^{\dagger}+\left(\begin{array}{ccc}
a(x, E) & 0 & 0 \\
0 & 0 & 0 \\
0 & 0 & 0
\end{array}\right)\right] .
\end{aligned}
$$

To the lowest order of perturbation, (6) can be solved by [11]

$$
S(x) \simeq S^{0}(x)+S^{\prime}(x),
$$

\footnotetext{
${ }^{2}$ We shall use $L$ to denote the baseline with respect to a measurement, $x$ to denote any intermediate length and $s$ for a dummy variable in any integration being performed upto $x$.
} 
with

$$
\begin{aligned}
S^{0}(x) & =e^{-i x H_{0}} \\
S^{\prime}(x) & =e^{-i x H_{0}}(-i) \int_{0}^{x} d s e^{i s H^{0}} H^{\prime}(s) e^{-i s H^{0}} .
\end{aligned}
$$

We turn now to matrix elements in the flavour basis and work in the approximations $\Delta m_{21}^{2} \ll$ $\left|\Delta m_{32}^{2}\right|$ and $|a(S, E)| \ll\left|\Delta m_{32}^{2}\right|$. First, it is convenient to define

$$
g(v) \equiv \frac{1}{4} \Delta m_{31}^{2} v
$$

Then we can explicitly write

$$
\begin{aligned}
S^{0}(x)_{\beta \alpha} & =\delta_{\beta \alpha}+U_{\beta 3} U_{\alpha 3}^{\star}\left[e^{-i g(2 x / E)}-1\right] \\
& =\delta_{\beta \alpha}-2 i U_{\beta 3} U_{\alpha 3}^{\star} e^{-i g(x / E)} \sin [g(x / E)] \\
& \equiv A_{\beta \alpha}(x / E)
\end{aligned}
$$

for the unperturbed part. The lowest order expression for the perturbed part

$$
S^{\prime}(x)_{\beta \alpha}=-i \int_{0}^{x} d s\left[e^{-i(x-s) H_{0}}\right]_{\beta \gamma}\left[H^{\prime}(s)\right]_{\gamma \delta}\left[e^{-s H_{0}}\right]_{\delta \alpha}
$$

can be rewritten, on using (7a), as

$$
\begin{aligned}
S^{\prime}(x)_{\beta \alpha}= & \left.-i \int_{0}^{x} d s U_{\beta i} \exp [-i \operatorname{diag}\{0,0, g(2(x-s) / E))\}\right]_{i i} U_{\gamma i}^{\star} \\
& {\left[H^{\prime}(s)\right]_{\gamma \delta} U_{\delta j} \exp [-i \operatorname{diag}\{0,0, g(2 s / E)\}]_{j j} U_{\alpha j}^{\star} . }
\end{aligned}
$$

In the RHS of $(12), H^{\prime}(s)$ has two additive parts, one constant and one depending on $s$ :

$$
H^{\prime}(s)=H^{1}+H^{a}(s)
$$

with

$$
\begin{aligned}
H^{1} & =\frac{1}{2 E} U\left(\begin{array}{ccc}
0 & 0 & 0 \\
0 & \Delta m_{21}^{2} & 0 \\
0 & 0 & 0
\end{array}\right) U^{\dagger}, \\
H^{a} & =\frac{1}{2 E}\left(\begin{array}{ccc}
a(s, E) & 0 & 0 \\
0 & 0 & 0 \\
0 & 0 & 0
\end{array}\right) .
\end{aligned}
$$

Correspondingly, we can take

$$
S^{\prime}(x)_{\beta \alpha}=S^{1}(x)_{\beta \alpha}+S^{a}(x)_{\beta \alpha},
$$


where $H^{1}$ contributes to $S^{1}$ and $H^{a}$ to $S^{a}$. Since $H^{1}$ does not depend on s, we may write

$$
\begin{aligned}
S^{1}(x)_{\beta \alpha}=- & -i U_{\beta i} U_{\gamma i}^{*}\left(H^{1}\right)_{\gamma \delta} U_{\delta j} U_{\alpha j}^{*} . \\
& \int_{0}^{x} d s \exp [-i \operatorname{diag}\{0,0, g(2(x-s) / E)\}]_{i i} \exp [-i \operatorname{diag}\{0,0, g(2 s / E)\}]_{j j} \\
= & -i \frac{\Delta m_{21}^{2}}{2 E} U_{\beta 2} U_{\alpha 2}^{*} \equiv-i B_{\beta \alpha}(x / E) \Delta m_{21}^{2},
\end{aligned}
$$

where we have used the identity

$$
U_{\gamma i}^{*}\left[H^{1}\right]_{\gamma \delta} U_{\delta j}=\frac{\Delta m_{21}^{2}}{2 E} \delta_{i 2} \delta_{j 2} .
$$

Turning to $S^{a}(x)_{\beta \alpha}$, it is convenient to use the result

$$
U_{\gamma i}^{*}\left[H^{a}(s)\right]_{\gamma \delta} U_{\delta j}=\frac{a(s, E)}{2 E} U_{1 i}^{*} U_{1 j} .
$$

The employment of (17) enables us to write $S^{a}(x)_{\beta \alpha}$ as

$$
\begin{aligned}
S_{\beta \alpha}^{a}(x, E) & =-\frac{i}{2 E} U_{\beta i} U_{\alpha j}^{*} U_{1 i}^{*} U_{1 j} \int_{0}^{x} d s \exp \left[-i g\left\{\frac{(2 x-2 s) \delta_{i 3}+2 s \delta_{j 3}}{E}\right\}\right] a(s, E), \\
& =-\frac{i}{2 E} U_{\beta i} U_{\alpha j}^{*} U_{1 i}^{*} U_{1 j} \Gamma_{i j}^{a}(x, E)
\end{aligned}
$$

with

$$
\begin{aligned}
\Gamma_{i j}^{a}(x, E) & =\delta_{i 3} \delta_{j 3} e^{-i g(2 x / E)} \int_{0}^{x} d s a(s, E)+\left(1-\delta_{i 3}\right)\left(1-\delta_{j 3}\right) \int_{0}^{x} d s a(s, E) \\
& +\left(1-\delta_{i 3}\right) \delta_{j 3} \int_{0}^{x} d s a(s, E) e^{-i g(2 s / E)}+\delta_{i 3}\left(1-\delta_{j 3}\right) \int_{0}^{x} d s a(s, E) e^{-i g(2(x-s) / E)} .
\end{aligned}
$$

On using (19) in (18), we obtain

$$
\begin{aligned}
S_{\beta \alpha}^{a}(x, E)= & -\frac{i}{2 E} \delta_{\beta 1} \delta_{1 \alpha} \int_{0}^{x} d s a(S, E) \\
& -\frac{1}{E} \delta_{1 \alpha} U_{\beta 3} U_{13}^{*} \int_{0}^{x} d s a(s, E) e^{-i[g(x / E)-g(s / E)]} \sin [g(x / E)-g(s / E)] \\
& -\frac{1}{E} \delta_{\beta 1} U_{\alpha 3}^{*} U_{13} \int_{0}^{x} d s a(s, E) e^{-i g(s / E)} \sin [g(s / E)] \\
& -\frac{i}{2 E} U_{\alpha 3}^{*} U_{\beta 3}\left|U_{13}\right|^{2} e^{-i g(x / E)} R(x, E) \\
\equiv & -i G_{\beta \alpha}^{a}(x, E) .
\end{aligned}
$$

In (20) we have introduced the real function $R(x, E)$ :

$$
\begin{aligned}
R(x, E) & \left.\equiv e^{-i g(x / E)} \int_{0}^{x} d s a(s, E)\left\{1-e^{i g(2 s / E)}\right\}+e^{i g(x / E)} \int_{0}^{x} d s a(s, E)\left\{1-e^{-i g(2 s / E)}\right)\right\}, \\
& \equiv 2 \int_{0}^{x} d s a(s, E)[\cos g(x / E)-\cos \{g(x / E)-g(2 s / E)\}] .
\end{aligned}
$$


Note that $R(x, E)$ becomes a function of $x / E$ for a constant $\rho(x)$, not otherwise.

Finally, the flavour matrix element of the evolution operator $S_{\beta \alpha}=S_{\beta \alpha}^{0}+S_{\beta \alpha}^{1}+S_{\beta \alpha}^{a}$ can be expressed as

$$
S_{\beta \alpha}(x, E)=A_{\beta \alpha}(x / E)-i B_{\beta \alpha}(x / E) \Delta m_{21}^{2}-i G_{\beta \alpha}^{a}(x, E),
$$

where $A_{\beta \alpha}(x / E), B_{\beta \alpha}(x / E)$ and $G_{\beta \alpha}^{a}(x, E)$ are given by (11), (16) and (20) respectively. We can now calculate the transition probability $P_{\beta \alpha}$ to the lowest order in $a$ and $\Delta m_{21}^{2}$ in the approximations $\Delta m_{21}^{2} \ll\left|\Delta m_{31}^{2}\right|,|a(s, E)| \ll\left|\Delta m_{31}^{2}\right|$ stated already. We obtain

$$
P_{\beta \alpha}=P_{\beta \alpha}^{0}+P_{\beta \alpha}^{\alpha}
$$

with

$$
\begin{gathered}
P_{\beta \alpha}^{0}=A_{\beta \alpha}^{*} A_{\beta \alpha}+2 \operatorname{Im}\left(A_{\beta \alpha}^{*} B_{\beta \alpha}\right), \\
P_{\beta \alpha}^{a}=2 \operatorname{Im}\left(A_{\beta \alpha}^{*} G_{\beta \alpha}^{a}\right),
\end{gathered}
$$

\section{The flavour survival probability $\nu_{\mu}(0) \rightarrow \nu_{\mu}(L)$}

Using (11), (16), (20) and choosing $\alpha=\beta=\mu$, we have

$$
\begin{aligned}
A_{\mu \mu}(x / E) & =1-2 i\left|U_{\mu 3}\right|^{2} e^{-i g(x / E)} \sin [g(x / E)], \\
B_{\mu \mu}(x / E) & =\left|U_{\mu 2}\right|^{2} \frac{x}{2 E}, \\
G_{\mu \mu}^{a}(x, E) & =\frac{\left|U_{e 3}\right|^{2}\left|U_{\mu 3}\right|^{2}}{2 E} e^{-i g(x / E)} R(x, E),
\end{aligned}
$$

with $R(x, E)$ as defined in $(21)$. In the matter free case, $a(s, E)=0$ and replacing $x$ by $L$ we obtain

$$
\begin{aligned}
P_{\mu \mu}^{0}(L / E) & =1-4\left|U_{\mu 3}\right|^{2} \sin ^{2}[g(L / E)] \\
& +4\left|U_{\mu 3}\right|^{4} \sin ^{2}[g(L / E)]+\frac{L}{E}\left|U_{\mu 2}\right|^{2}\left|U_{\mu 3}\right|^{2} \sin [2 g(L / E)] \Delta m_{21}^{2}
\end{aligned}
$$

If we consider this transition to be overwhelmingly driven by a two flavour oscillation, as done in the super-K analysis [6], we can ignore the third RHS term in (27) to see that the depletion $1-$ $P_{\mu \mu}^{0}(L, E)$, i.e. the flavour transformation and hence mixing for a muonic neutrino or antineutrino, would be maximal for $\left|U_{\mu 3}\right|=1 / \sqrt{2}$. Next, we consider propagation in matter and keep $a$ and hence $M(L)$. Then we have

$$
P_{\mu \mu}(L, E)=P_{\mu \mu}^{0}(L / E)+P_{\mu \mu}^{a}(L, E)
$$

where

$$
P_{\mu \mu}^{a}(L, E)=\frac{\left|U_{e 3}\right|^{2}\left|U_{\mu 3}\right|^{2}}{E}\left(2\left|U_{\mu 3}\right|^{2}-1\right) \sin [g(L / E)] R(L, E) .
$$


The asymmetry $\Delta P_{\mu \mu}(L, E)$, defined in (1), is thus

$$
\begin{aligned}
\Delta P_{\mu \mu}(L, E) & =\frac{\left|U_{e 3}\right|^{2}\left|U_{\mu 3}\right|^{2}}{E}\left(2\left|U_{\mu 3}\right|^{2}-1\right) \sin [g(L / E)]\left[R^{+}(L, E)-R^{-}(L, E)\right] \\
& =\frac{2\left|U_{e 3}\right|^{2}\left|U_{\mu 3}\right|^{2}}{E}\left(2\left|U_{\mu 3}\right|^{2}-1\right) \sin [g(L / E)] R^{+}(L, E) .
\end{aligned}
$$

In $(30) R^{ \pm}(L, E)$ is obtained from $(21)$ by using $a(s, E) \equiv \pm|a(s, E)|$ respectively so that $R^{-}(L, E)=$ $-R^{+}(L, E)$. Thus we see that the matter effect contribution to the muonic flavour survival probability $P_{\mu \mu}(x)$ vanishes if $\left|U_{\mu 3}\right|=1 / \sqrt{2}$. Moreover, even in matter and to the lowest order of perturbation, any nonzero asymmetry $\Delta P_{\mu \mu}$, detected from the muon (anti) neutrino flavour survival probabilities $P\left(\stackrel{(-)}{\nu_{\mu}} \rightarrow \stackrel{(-)}{\nu_{\mu}}\right)$ will signal a deviation from the condition for the strictly maximal mixing of atmospheric neutrinos at super-K. This can be used in future to sensitively probe any deviation of $\left|U_{\mu 3}\right|$ from its maximal value $1 / \sqrt{2}$.

\section{General oscillation probability in matter: $\nu_{\alpha} \rightarrow \nu_{\beta}$}

From (24) and (26a,b) we have

$$
\begin{aligned}
P_{\beta \alpha}^{0}(L / E) \simeq & \delta_{\beta \alpha}\left[1-4\left|U_{\alpha 3}\right|^{2} \sin ^{2}[g(L / E)]\right]+4\left|U_{\alpha 3}\right|^{2}\left|U_{\beta 3}\right|^{2} \sin ^{2}[g(L / E)] \\
& +\frac{L \Delta m_{21}^{2}}{E}\left[\operatorname{Re}\left(U_{\alpha 3}^{*} U_{\beta 3} U_{\alpha 2} U_{\beta 2}^{*}\right) \sin [2 g(L / E)]\right. \\
& \left.-2 \operatorname{Im}\left(U_{\alpha 3}^{*} U_{\beta 3} U_{\alpha 2} U_{\beta 2}^{*}\right) \sin ^{2}[g(L / E)]\right]
\end{aligned}
$$

while (25) and (26a,c) lead us to the expression

$$
\begin{aligned}
P_{\beta \alpha}^{a}(L, E) \simeq & 2 \operatorname{Im}\left[A_{\beta \alpha}^{*}(L / E) G_{\beta \alpha}^{a}(L, E)\right] \\
= & \delta_{\beta \alpha}\left\{\frac{1}{E} \delta_{1 \alpha}\left|U_{\alpha 3}\right|^{2} \sin ^{2}[g(L / E)] \int_{0}^{L} d s a(s, E) \sin [g(L / E)-g(s / E)]\right. \\
& \left.-\frac{1}{E}\left|U_{\alpha 3}\right|^{2}\left|U_{e 3}\right|^{2} \sin [g(L / E)] R(L, E)\right\} \\
& +\frac{1}{E}\left|U_{\alpha 3}\right|^{2}\left|U_{\beta 3}\right|^{2}\left\{2\left|U_{e 3}\right|^{2}\left(\delta_{1 \alpha}+\delta_{\beta 1}\right) \sin [g(L / E)]\right\} R(L, E),
\end{aligned}
$$

with $R(L, E)$ substituted from (21). 
We can now discuss two distinct cases.

Case 1: $\alpha=\beta$

- $\alpha=\beta \neq e$

In this case the matter independent and matter dependent transition probabilities, cf.

$$
\begin{aligned}
& P_{\alpha \alpha}^{0}(L / E)=1-4\left|U_{\alpha 3}\right|^{2}\left(1-\left|U_{\alpha 3}\right|^{2}\right) \sin ^{2}[g(L / E)]+\frac{\Delta m_{21}^{2} L}{E}\left|U_{\alpha 2}\right|^{2}\left|U_{\alpha 3}\right|^{2} \sin [2 g(L / E)], \\
& P_{\alpha \alpha}^{a}(L, E)=\frac{1}{E}\left|U_{\alpha 3}\right|^{2}\left|U_{e 3}\right|^{2}\left(2\left|U_{\alpha 3}\right|^{2}-1\right) \sin [g(L / E)] R(L, E) .
\end{aligned}
$$

- $\alpha=\beta=e$

The expression for $P_{e e}^{0}(L)$ is the same as the RHS of (33a) with $\alpha=e$, but the matter dependent part is

$$
\begin{aligned}
P_{e e}^{a}(L, E)= & \frac{1}{E}\left|U_{e 3}\right|^{2} \sin ^{2}[g(L / E)] \int_{0}^{L} d s a(s, E) \sin [g(L / E) \\
& -k(s / E) / 2]-\frac{1}{E}\left|U_{e 3}\right|^{4} \sin [g(L / E)] R(L, E) \\
& +\frac{2}{E}\left|U_{e 3}\right|^{4}\left(\left|U_{e 3}\right|^{2}-1\right) \sin [g(L / E)] R(L, E) .
\end{aligned}
$$

Case 2: $\alpha \neq \beta$

Now the matter independent transition probability is given from (31) by

$$
P_{\beta \alpha}^{0}(L / E)=4\left|U_{\beta 3}\right|^{2}\left|U_{\alpha 3}\right|^{2} \sin ^{2}[g(L / E)]-\frac{2 \Delta m_{21}^{2} L}{E}\left\{\operatorname{Im} \xi \sin ^{2}[g(L / E)]-\frac{1}{2} \operatorname{Re} \xi \sin [2 g(L / E)]\right\},
$$

with

$$
\xi \equiv U_{\beta 2}^{*} U_{\beta 3} U_{\alpha 2} U_{\alpha 3}^{*} .
$$

For the matter dependent part, (32) leads to three cases.

- $\alpha \neq e, \beta \neq e$

$$
P_{\beta \alpha}^{a}(L, E)=\frac{2}{E}\left|U_{\alpha 3}\right|^{2}\left|U_{\beta 3}\right|^{2}\left|U_{e 3}\right|^{2} \sin [g(L / E)] R(L, E) .
$$

- $\alpha \neq e, \beta=e$

$$
P_{e \alpha}^{a}(L, E)=\frac{1}{E}\left|U_{\alpha 3}\right|^{2}\left|U_{e 3}\right|^{2}\left(2\left|U_{e 3}\right|^{2}-1\right) \sin [g(L / E)] R(L, E) .
$$

- $\alpha=e, \beta \neq e$

$$
P_{\beta e}^{a}(L, E)=\frac{1}{E}\left|U_{e 3}\right|^{2}\left|U_{\beta 3}\right|^{2}\left(2\left|U_{e 3}\right|^{2}-1\right) \sin [g(L / E)] R(L, E) .
$$


Both the CP violating part, proportional to $\operatorname{Im} \xi$ in (35), and the matter dependent part $P_{\beta \alpha}^{a}$ change sign when one goes from neutrinos and antineutrinos. However, the former is a function of $x / E$, while the latter involves both $x / E$ and $x$ in an unknown way for a general earth matter density function $\rho(x)=\left(2 \sqrt{2} G_{F} N_{e} E\right)^{-1} a(x, E)$. Only for $\rho(x)=$ constant, can all the transition probabilities become functions of $L / E$ and the matter dependent part can be eliminated, to the lowest order of perturbation, by [9] taking $L_{1}\left(L_{2}-L_{1}\right)^{-1}\left[\Delta P_{\beta \alpha}\left(L_{2}\right)-\Delta P_{\beta \alpha}\left(L_{1}\right)\right]_{L / E \text { fixed }}$, but this procedure is in general invalid for a spatially varying $a(x, E)$.

\section{$5 \quad$ Numerical estimate of matter induced asymmetry}

In this section we present some numerical results for the matter contribution to the transition probabilities using the formalism developed in this paper. We choose realistic neutrino beam energies and both realistic and notional detector baselines and discuss the conditions for the validity of our perturbative calculation. For numerical studies we have used the Preliminary Reference earth Model(PREM) [13] and another earth model - ak135-F [19]. For the validity of perturbation theory for all intermediate values of $s$ we must satisfy the conditions

$$
\begin{array}{cc}
\frac{a(s, E) s}{4 E} & <\frac{\Delta m_{31}^{2} s}{4 E} \\
\frac{a(s, E) s}{2 E}<<1 ; & \frac{\Delta m_{21}^{2} s}{2 E}<<1 \\
\sin ^{2}\left(\frac{\Delta m_{21}^{2} s}{4 E}\right) & <<\sin ^{2}\left(\frac{\Delta m_{31}^{2} s}{4 E}\right),
\end{array}
$$

where $0 \leq s \leq L$. Note that conditions in eqns (41) are required for the expansion of $S(x)$ in Section 2. Neutrino oscillation experiments either look for disappearance of the initial neutrino beam or for the appearance of a different flavour in the final neutrino beam at the detector. Both these effects are maximal when $\sin ^{2}\left(\Delta m_{31}^{2} L / 4 E\right) \sim 1$ corresponding to a "peak" in the transition probability. The Super-Kamiokande atmospheric neutrino data demands a $\Delta m_{31}^{2} \sim 3 \times 10^{-3} \mathrm{eV}^{2}$ [6] while the mass squared difference associated with the solar neutrino oscillation has now been confirmed to be around $\Delta m_{21}^{2} \sim 7 \times 10^{-5} \mathrm{eV}^{2}$ by the KamLAND experiment [5]. Thus for all realistic experimental scenarios where the beam energy is tuned to an oscillation maximum for $\Delta m_{31}^{2}$, oscillations driven by the solar scale is always subdominant compared to those driven by the atmospheric scale, i.e. the condition (42) and the second of conditions (41) are satisfied. For the validity of our approximation (42) we will therefore always confine ourselves to values of

$$
\frac{L / K m}{E / G e V} \sim O\left(10^{2}-10^{3}\right)
$$

To check our other approximation concerning the matter potential we present in Figure 1 the comparison of the strength of the matter term vis-a-vis the term involving $\Delta m_{31}^{2}$. The solid line in the left-hand panel shows $\overline{a_{L}} L / 4 E$, where $\overline{a_{L}}$ is the "average" matter potential for a given neutrino baseline $L$ defined as

$$
\overline{a_{L}}=\frac{1}{L} \int_{0}^{L} a(s, E) d s
$$




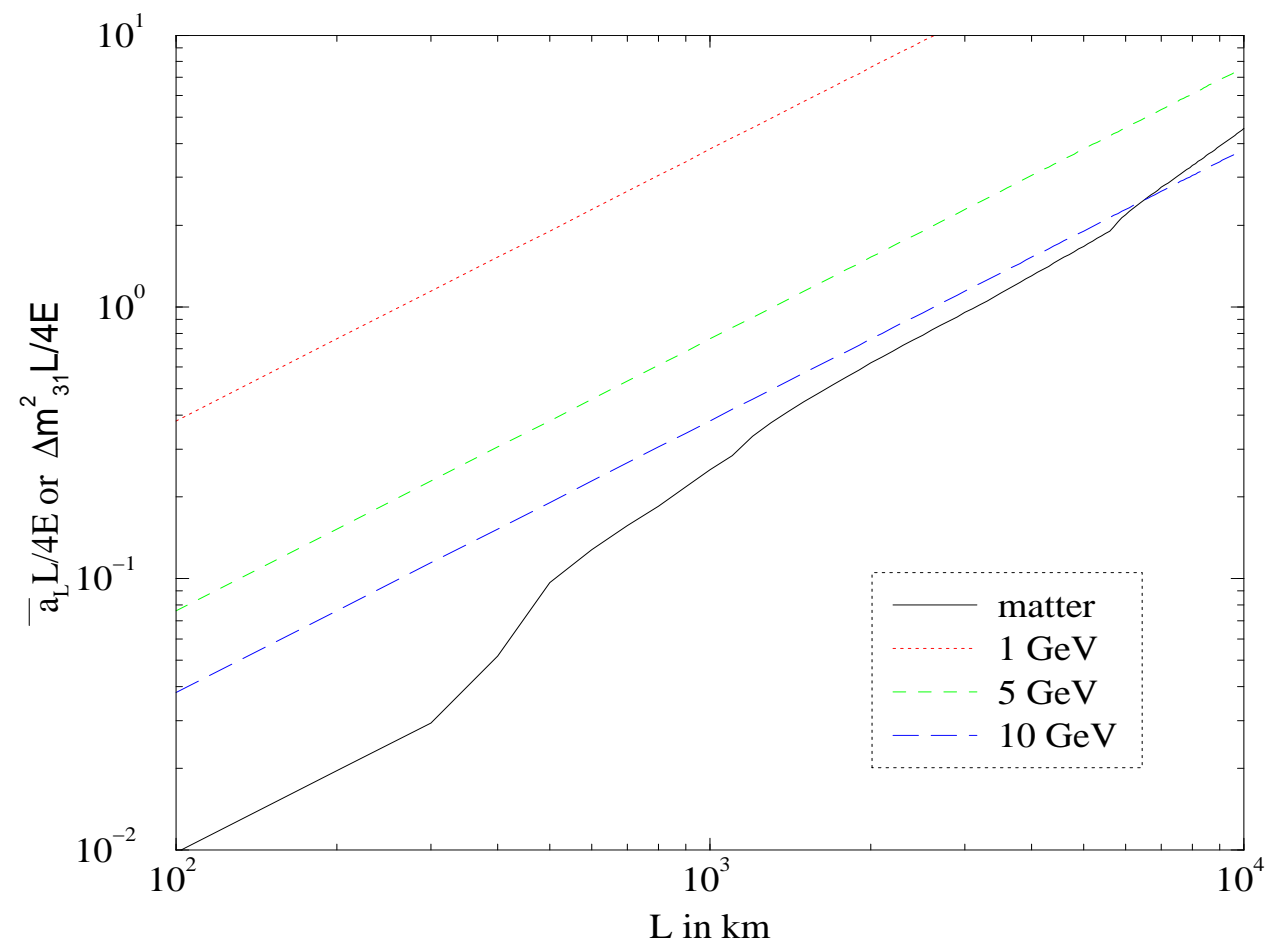

Figure 1: The comparison of the matter term against the $\Delta m_{31}^{2}$ term as a function of the neutrino baseline and for different neutrino energies. The matter term is independent of energy and is shown by the black solid line.

For each experimental baseline $L, \overline{a_{L}}$ is computed using the PREM model [13]. Since $a(s, E)$ goes linearly with the neutrino energy $E$ (cf. Eq.(2)), the matter potential term $\overline{a_{L}} L / 4 E$ is independent of the energy of the neutrino beam. It is only a function of the average matter density and hence of the baseline $L$. The dotted, dashed and long-dashed lines show $\Delta m_{31}^{2} L / 4 E$ for $E=1,5$ and $10 \mathrm{GeV}$ respectively. We note that for neutrinos with $E=3 \mathrm{GeV}$ the approximation given in Eq. (40) works very well for all baselines shown. However for neutrino energies equal to or in excess of $10 \mathrm{GeV}$ our perturbative expansion for the matter term works only for smaller $L$ and breaks down at higher baselines.

In Table 1 we give a summary of the magnitude of $\Delta P^{a}$ for some specific cases. In Figure 2 we show $\Delta P^{a}$ as a function of the baseline length $L$ for the $\nu_{\mu} \rightarrow \nu_{e}$ transition and with $E=3 \mathrm{GeV}$. We have checked that very similar behaviour is exhibited by $\Delta P^{a}$ for the transitions $\nu_{e} \rightarrow \nu_{\tau}$ and $\nu_{\mu} \rightarrow \nu_{\tau}$. The solid and the dashed lines show the $\Delta P^{a}$ obtained using our formalism for a varying density matter profile for the earth. The dashed line is for the PREM model [13] while the solid line corresponds to the ak135-F model [19] for the earth matter profile. The dotted line gives the corresponding values for a constant density earth with $\rho=3.28 \mathrm{gm} / \mathrm{cc}$. The values of parameters used for generating the figure and the table are shown in the captions. Comparison of the solid and/or dashed lines with the constant density dotted line shows that the variation in the density profile can lead to a change in the oscillation probability. Even at $L=3000 \mathrm{~km}$, we note about 


\begin{tabular}{|c|c|c|c|c|c|c|}
\hline $\mathrm{E} / \mathrm{GeV}$ & $U_{e 3}$ & $\left|\Delta P_{\tau \mu}^{a}(732)\right|$ & $\left|\Delta P_{e \mu}^{a}(732)\right|$ & $\left|\Delta P_{\mu e}^{a}(732)\right|$ & $\left|\Delta P_{\tau e}^{a}(732)\right|$ & $R$ in $\mathrm{GeV}$ \\
\hline 1 & 0.001 & $5.32 \times 10^{-7}$ & $5.32 \times 10^{-7}$ & $5.32 \times 10^{-7}$ & $5.32 \times 10^{-7}$ & -1.54 \\
1 & 0.01 & $5.32 \times 10^{-5}$ & $5.32 \times 10^{-5}$ & $5.32 \times 10^{-5}$ & $5.32 \times 10^{-5}$ & -1.54 \\
1 & 0.1 & $5.21 \times 10^{-3}$ & $5.21 \times 10^{-3}$ & $5.21 \times 10^{-3}$ & $5.11 \times 10^{-3}$ & -1.54 \\
\hline $\mathrm{E} / \mathrm{GeV}$ & $U_{e 3}$ & $\left|\Delta P_{\tau \mu}^{a}(2500)\right|$ & $\left|\Delta P_{e \mu}^{a}(2500)\right|$ & $\left|\Delta P_{\mu e}^{a}(2500)\right|$ & $\left|\Delta P_{\tau e}^{a}(2500)\right|$ & $R$ in $\mathrm{GeV}$ \\
\hline 3 & 0.001 & $2.16 \times 10^{-7}$ & $2.16 \times 10^{-7}$ & $2.16 \times 10^{-7}$ & $2.16 \times 10^{-7}$ & -19.42 \\
3 & 0.01 & $2.16 \times 10^{-5}$ & $2.16 \times 10^{-5}$ & $2.16 \times 10^{-5}$ & $2.16 \times 10^{-5}$ & -19.42 \\
3 & 0.1 & $2.12 \times 10^{-3}$ & $2.12 \times 10^{-3}$ & $2.12 \times 10^{-3}$ & $2.08 \times 10^{-3}$ & -19.42 \\
\hline $\mathrm{E} / \mathrm{GeV}$ & $U_{e 3}$ & $\left|\Delta P_{\tau \mu}^{a}(3000)\right|$ & $\left|\Delta P_{e \mu}^{a}(3000)\right|$ & $\left|\Delta P_{\mu e}^{a}(3000)\right|$ & $\left|\Delta P_{\tau e}^{a}(3000)\right|$ & $R$ in $\mathrm{GeV}$ \\
\hline 5 & 0.001 & $5.72 \times 10^{-6}$ & $5.72 \times 10^{-6}$ & $5.72 \times 10^{-6}$ & $5.72 \times 10^{-6}$ & -37.91 \\
5 & 0.01 & $5.72 \times 10^{-4}$ & $5.72 \times 10^{-4}$ & $5.72 \times 10^{-4}$ & $5.72 \times 10^{-4}$ & -37.91 \\
5 & 0.1 & $5.61 \times 10^{-2}$ & $5.61 \times 10^{-2}$ & $5.61 \times 10^{-2}$ & $5.50 \times 10^{-2}$ & -37.91 \\
\hline $\mathrm{E} / \mathrm{GeV}$ & $U_{e 3}$ & $\left|\Delta P_{\tau \mu}^{a}(3500)\right|$ & $\left|\Delta P_{e \mu}^{a}(3500)\right|$ & $\left|\Delta P_{\mu e}^{a}(3500)\right|$ & $\left|\Delta P_{\tau e}^{a}(3500)\right|$ & $R$ in GeV \\
\hline 7 & 0.001 & $7.03 \times 10^{-6}$ & $7.03 \times 10^{-6}$ & $7.03 \times 10^{-6}$ & $7.03 \times 10^{-6}$ & -52.10 \\
7 & 0.01 & $7.03 \times 10^{-4}$ & $7.03 \times 10^{-4}$ & $7.03 \times 10^{-4}$ & $7.03 \times 10^{-4}$ & -52.10 \\
7 & 0.1 & $6.89 \times 10^{-2}$ & $6.89 \times 10^{-2}$ & $6.89 \times 10^{-2}$ & $6.75 \times 10^{-2}$ & -52.10 \\
\hline
\end{tabular}

Table 1: The magnitude of $\Delta P^{a}$ for some specific values of $E$ and $L$ and for the different oscillation channels. Values of $L$ in $\mathrm{kms}$ are shown in parantheses after $\Delta P^{a}$. Also shown is the value of the function $R(L, E)$ defined in Eq.(32). We have taken $\Delta m_{31}^{2}=3 \times 10^{-3} \mathrm{eV}^{2}, \Delta m_{21}^{2}=7 \times 10^{-5} \mathrm{eV}^{2}$ and $\left|U_{\mu 3}\right|^{2}=0.5$. We present results for three different values of $\left|U_{e 3}\right|$.

$10 \%$ difference in $\Delta P^{a}$ between the constant density case and the case corresponding to PREM or ak135-F. At smaller $L$ we note a small difference between $\Delta P^{a}$ corresponding to PREM and ak135-F, which just reflects the fact that ak135-F has more density fluctuation at smaller $L$ than $\mathrm{PREM}^{3}$. We see that the effect of density variation begins to be significant for $L>5000 \mathrm{kms}$. A caveat is that out formulae are not quantitatively reliable if $L$ and $E$ are such that either (40) or the first of conditions (41) breaks down. (The second condition of (41) is always safely obeyed). Such is evidently not the case in most of Figure 1 . Work is in progress to improve upon the first order perturbation theory in $a(s, E)$ so that the dependence on these conditions is reduced.

\section{Conclusions}

With the confirmation of neutrino flavour oscillations both in the atmospheric as well as the solar neutrino sectors, the focus now has shifted to the precise determination of the oscillation parameters involved. This will be possible in the currently planned and future long and very long

\footnotetext{
${ }^{3}$ It has been pointed out in [17] that even the so called realistic earth models like PREM and ak135-F neglect the local density fluctuations which can have an impact on the final oscillation probability and hence on the CP sensitivity of a specific experiment. However our expressions for the oscillation probabilities in varying density matter are completely general and can be applied to any earth matter density profile, local variations notwithstanding.
} 


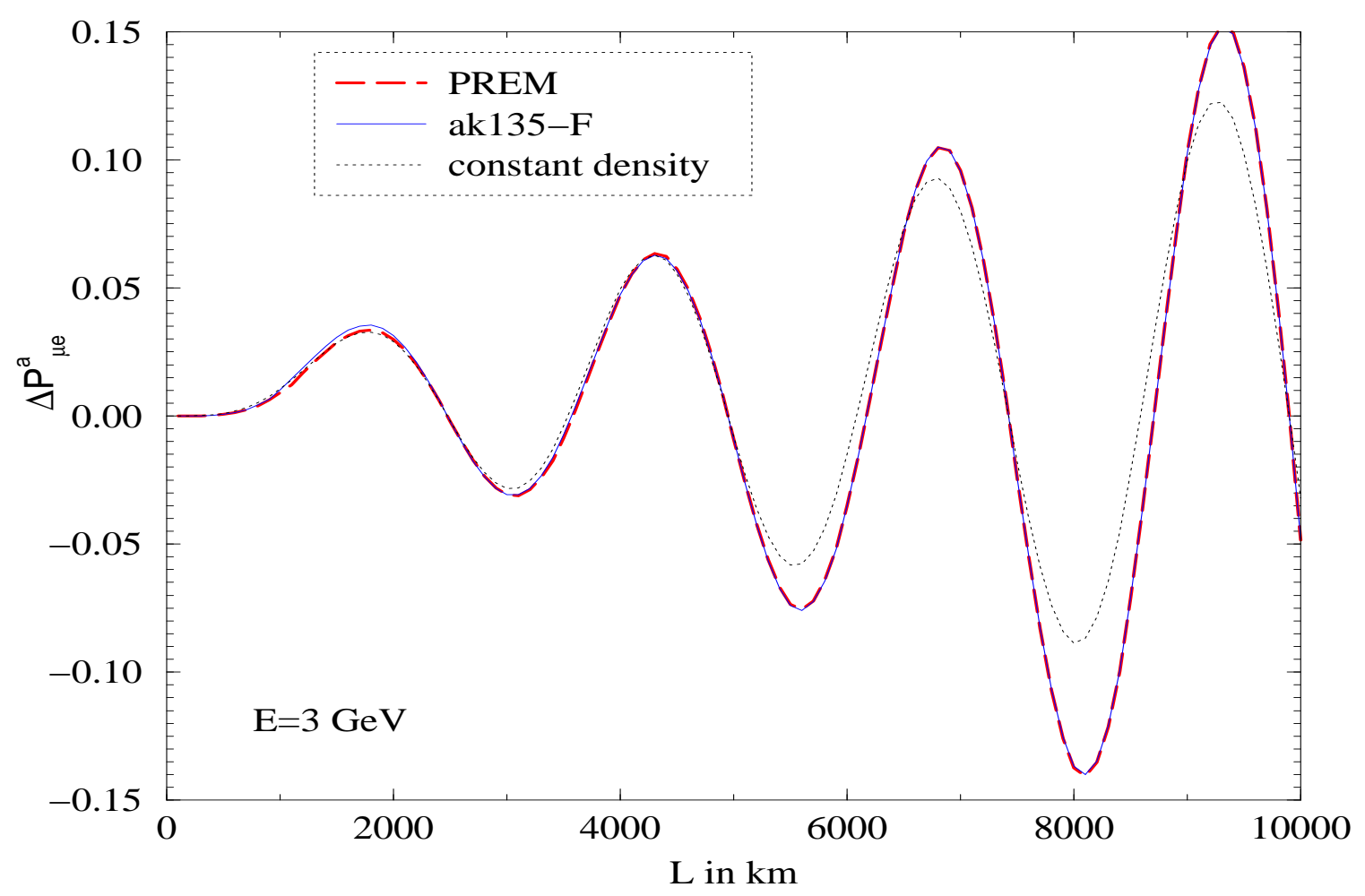

Figure 2: The CP asymmetry induced by matter effect $\left(\Delta P^{a}\right)$ as a function of the baseline $L$ for the $\nu_{\mu} \rightarrow \nu_{e}$ oscillation channel with $E=3 \mathrm{GeV}$. The dashed line and the solid line give the $\Delta P^{a}$ calculated using the PREM model and the ak135-F model respectively, while the dotted line is for a constant density earth with $\rho=3.28 \mathrm{gm} / \mathrm{cc}$. We have taken $\Delta m_{31}^{2}=3 \times 10^{-3} \mathrm{eV}^{2}$, $\Delta m_{21}^{2}=7 \times 10^{-5} \mathrm{eV}^{2},\left|U_{\mu 3}\right|^{2}=0.5$ and $\left|U_{e 3}\right|^{2}=0.01$.

baseline experiments involving conventional superbeams and neutrino factories. The determination of $\mathrm{CP}$ violation in the lepton sector and measurement of the $\mathrm{CP}$ phase will be the most interesting as well as the most challenging goal of these experiments. The matter effect induces a "fake" CP asymmetry even if there is no intrinsic $\mathrm{CP}$ violation in the neutrino sector. Thus a knowledge of matter effect is extremely important in all neutrino $\mathrm{CP}$ violation studies. The matter effect also helps in ascertaining the sign of the atmospheric neutrino mass squared difference $\Delta m_{31}^{2}$ which has a bearing on the neutrino mass hierarchy with profound theoretical and phenomenological implications. The effect of earth matter on the survival and transition probabilities were studied earlier and ways to disentangle the "real" CP from the "fake" CP due to matter were discussed. However most of these studies assumed a constant density of the earth matter.

In this paper we have used the evolution operator formalism to derive the most general lowest order perturbative expressions for neutrino flavour survival and transition probabilities in varying density matter. We have worked in a perturbative scheme, where the oscillation driven by $\Delta m_{31}^{2}$ is assumed to be much larger than those driven by $\Delta m_{21}^{2}$ and the matter potential $a(s, E)$. We have shown to the lowest nontrivial order that for a maximal mixing in the $\nu_{\mu}-\nu_{\tau}$ sector, the 
matter effect in the survival probability $P_{\mu \mu}$ vanishes identically. We have made numerical checks for the validity of our perturbation approximation and conclude that as long as $E \lesssim 10 \mathrm{GeV}$, our approximation holds, at least upto a baseline of $L \sim 4000 \mathrm{~km}$. Finally, we have compared the results obtained with the PREM and ak135-F density profiles with that for a constant density earth matter. Work is in progress to extend the range of validity of our theory to longer baselines.

\section{Acknowledgements}

B.B. acknowledges hospitality of Department of Theoretical Physics, TIFR and of Theory Division, CERN. S.C. thanks S.T. Petcov for discussions. P.R. is indebted to Theory Group, SLAC and to Santa Cruz Institute for Particle Physics, UCSC, for their hospitality.

\section{References}

[1] N. Cabibbo, Phys. Lett. B72, 333 (1978); V. D. Barger, K. Whistant and R. J. N. Philips, Phys. Rev. Lett. 452084 (1980); S. Pakvasa, Proc. XXth Intl. Conf. on High Energy Physics, Madison (1980), p 1164; S. M. Bilenky and F. Niedermeyer, Sov. J. Nucl. Phys. 34, 606 (1981) [Yad. Fiz. 34, 1091 (1981)].

[2] A. de Rujula, M.B. Gavela and P. Hernandez, Nucl. Phys. B547, 21 (1999) [arXiv: hepph/9911390]. C. Albright et al., arXiv:hep-ex/0008064. M. Apollonio et al., arXiv:hepph/0210192. Y. Itow et al., arXiv:hep-ex/0106019. D. Ayres et al., arXiv:hep-ex/0210005. M. Lindner, arXiv:hep-ph/0209083; K. Dick, M. Freund, M. Lindner and A. Romanino, Nucl. Phys. B 562, 29 (1999) [arXiv:hep-ph/9903308]; A. Cervera, A. Donini, M. B. Gavela, J. J. Gomez Cadenas, P. Hernandez, O. Mena and S. Rigolin, Nucl. Phys. B 579, 17 (2000) [Erratum-ibid. B 593, 731 (2001)] [arXiv:hep-ph/0002108]; V. Barger, D. Marfatia and K. Whisnant, Phys. Rev. D 65, 073023 (2002) [arXiv:hep-ph/0112119] S. Geer, arXiv:hepph/0210113; O. Yasuda, arXiv:hep-ph/0209127.

[3] M. Fukugita and T. Yanagida, Phys. Lett. B175, 45 (1986); G. Branco, T. Morozumi, B. Nobre and M. N. Rebelo, Nucl. Phys. B617, 475 (2001); A. Joshipura, E. Pascos and W. Rodejohann, JHEP 0108, 029 (2001); W. Buchmüller and D. Wyler, Phys. Lett. B521, 291 (2001); T. Endoh, T. Morozumi and A. Purwanto, Nucl. Phys. Proc. Suppl. 111, 291 (2002); J. Ellis amd M. Raidal, Nucl. Phys. B 643, 229 (2002); S. Davidson and A. Ibarra, arXiv: hep-ph/0206304; G. C. Branco, R. Gonzalez Felipe, F. R. Joaquim and M. N. Rebelo, Nucl. Phys. Proc. Suppl. 111, 303 (2002); P. Frampton, S. Glashow and T. Yanagida, arXiv: hepph/0208157; T. Endoh, S. Kaneko, S. K. Kang, T. Morozumi and M. Tanimoto, Phys. Rev. Lett. 89231601 (2002).

[4] Q. R. Ahmad et al. [SNO Collaboration], Phys. Rev. Lett. 89, 011301 (2002) [arXiv:nuclex/0204008]; Q. R. Ahmad et al. [SNO Collaboration], Phys. Rev. Lett. 89, 011302 (2002) [arXiv:nucl-ex/0204009]; S. Fukuda et al. [Super-Kamiokande Collaboration], Phys. Lett. B 539, 179 (2002) [arXiv:hep-ex/0205075]; K. Eguchi et al. [KamLAND Collaboration], Phys. Rev. Lett. 90, 021802 (2003) [arXiv:hep-ex/0212021]. 
[5] A. Bandyopadhyay, S. Choubey, R. Gandhi, S. Goswami and D. P. Roy, arXiv:hepph/0212146; A. Bandyopadhyay, S. Choubey and S. Goswami, arXiv:hep-ph/0302243; V. Barger and D. Marfatia, arXiv:hep-ph/0212126; G. L. Fogli, E. Lisi, A. Marrone, D. Montanino, A. Palazzo and A. M. Rotunno, arXiv:hep-ph/0212127; M. Maltoni, T. Schwetz and J. W. Valle, arXiv:hep-ph/0212129; J. N. Bahcall, M. C. Gonzalez-Garcia and C. Pena-Garay, arXiv:hep-ph/0212147; P. C. de Holanda and A. Y. Smirnov, arXiv:hep-ph/0212270.

[6] Y. Fukuda et al., Phys. Rev. Lett. 81, 1562 (1998); ibid 85, 3999 (2001); T. Kajita and Y. Totsuka, Rev. Mod. Phys. 73, 85 (2001).

[7] M. Applonio et al., Phys. Lett. B466, 415 (1999); F. Boehm et al., Phys. rev. D64, 112001 (2001).

[8] J. J. Gomez-Cadenas et al., arXiv: hep-ph/0105297; M. Akoi, K. Hagiwara and N. Okamura, arXiv: hep-ph/0208223.

[9] I. Mocioiu and R. Shrock, Phys. Rev. D62, 0153017 (2001); I. Mocioiu and R. Shrock, JHEP 0111, 050 (2001); V. Barger, D. Marfatia and K. Whisnant, Phys. Rev. D65, 073023 (2002); T. Ohlsson and H. Snellman, Eur. Phys. J. C 20, 507 (2001).

[10] M. Freund, M. Lindner, S. T. Petcov and A. Romanino, Nucl. Phys. B 578, 27 (2000).

[11] J. Arafune, M. Koike and J. Sato, Phys. Rev. D56 3093 (1997); errtm. ibid. D60 119905 (1999).

[12] M. Freund and T. Ohlsson, Mod. Phys. Lett. A 15, 867 (2000).

[13] A.M. Dziewonski and D.L. Anderson, Phys. Earth Planet Inter. 25297 (1981); S.V. Panasyuk, Reference Earth Model (REM) webpage, http://cfauves5.harvrd.edu/lana/rem/index.html.

[14] M. Koike and J. Sato, Mod. Phys. Lett. A14 1297 (1999); T. Ota and J. Sato, Phys. Rev. D63 093004 (2000); B. Jacobsson, T. Ohlsson, H. Snellman and W. Winter, Phys. Lett. B 532, 259 (2002); B. Jacobsson, T. Ohlsson, H. Snellman and W. Winter, arXiv:hep-ph/0209147.

[15] T. Ota and J. Sato, arXiv: hep-ph/0211095.

[16] L. Y. Shan, B. L. Young and X. m. Zhang, Phys. Rev. D 66, 053012 (2002);

[17] L. Y. Shan, Y. F. Wang, C. G. Yang, X. Zhang, F. T. Liu and B. L. Young, arXiv:hep$\mathrm{ph} / 0303112$.

[18] K. Kimura, A. Takamura and H. Yokomakura, arXiv: hep-ph/0203099.

[19] B.N.L. Kennett, E.R. Engdahl and R. Buland, Geophys. J. Int., 122, 108, (1995); webpage http://wwwrses.anu.edu.au/seismology/ak135/intro.html. 